\title{
ADAPTIVE MULTI-ROBOT, MULTI-OPERATOR WORK SYSTEMS
}

\author{
Aaron C. Morris, Charles K. Smart, and Scott M. Thayer \\ Robotics Institute - Carnegie Mellon University \\ 5000 Forbes Avenue \\ Pittsburgh, PA 15213 \\ \{acmorr,cks,sthayer\}@cs.cmu.edu
}

\begin{abstract}
Unstructured and hostile environments impose great risk to exposed humans and present ideal domains for robotic forces; however, these dynamic environments pose considerable difficulty in autonomous multi-robot coordination, making a need for supervisory control paramount. This paper examines a three-phase approach that increases the robustness, reliability, and efficiency of humanmachine work systems by dynamically altering the soldier-robot control relationships as well as the effective autonomy manifested by each robot function in response to estimated cognitive loading (stress). This approach enables an adaptive command and control structure across a spectrum of force configurations.
\end{abstract}

Key words: human-machine work systems, command and control, supervisory control

\section{INTRODUCTION}

Unstructured and hostile environments impose great risk to exposed humans and present ideal domains for robotic applications; however, the conditions in such environments represent complex challenges for autonomous robot coordination. The uncertainties that exist in dynamic environments (i.e. dynamic obstacles, insufficient prior knowledge, etc.) can impede the attainment of robot objectives thereby reducing the dependability of such systems for military operations. Enhancements to autonomous multi-robot coordination are required for reliable performance in 
unstructured domains. The approach proposed in this paper utilizes human cognition and dynamically reconfigurable control structures to enhance the reliability of robot work systems.

The integration of human and machine has long been investigated and found to be mutually advantageous [1]. Recent research, [2] and [3], has developed adjustable autonomy to allow proportional alterations in robot autonomy so that humans can opportunistically inject guidance into autonomous command and control loops. Additionally, cognitive science, [4], has demonstrated successful modelling of human cognition so that situations of cognitive overload can be predicted and corrected before problems are manifested.

This paper outlines the merger of computational cognitive estimation with robot control theory to create a system that permits a small group of human operators the ability to manage a fleet of semi-autonomous robots within a spectrum of operational scenarios. This work is presented as a three-phase system (workload estimation, workload optimization, and workload mitigation) detailing the concept, mathematical background, and algorithm that form the linkage between human cognition and robot control.

\section{THE SYSTEM OVERVIEW}

An operator-robot work system embodies a set of tasks (T) assigned to a force of heterogeneous robotic units $(\mathbf{R})$ that are managed by a small crew of human operators $(\mathbf{H})$. Tasks represent a wide range of duties (e.g. moving from one point to another, mapping, etc.) that robotic units are expected to perform. Humans represent a finite resource of cognition to aid and assist robot action. Robots are represented as sets of capabilities, or subsystems (S), and the atomic actions, or functions $(\mathbf{F})$, that compose these subsystems. Modeling robots at a functional level allows (1) operators to enact control to specific actions providing a more efficient distribution of robot resources and (2) adaptive robot management since operator attention can be tuned upon to an optimized set of actions (Figure 1).

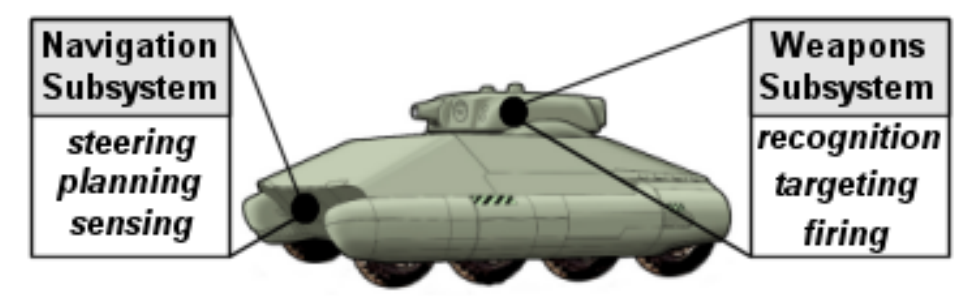

Figure 1 Decomposition of robot into subsystems and functions 
The mathematical representation of these components is $\mathbf{H}=\left\{h_{1}, h_{2}, \ldots, h_{M}\right\}$, $\mathbf{R}=\left\{r_{1}, r_{2}, \ldots, r_{N}\right\}$, and $\mathbf{T}=\left\{t_{1}, t_{2}, \ldots, t_{P}\right\}$ for $M$ operators, $N$ robots, and $P$ tasks, respectively. Furthermore, a robot is a set of subsystems (or a subset of all subsystems) $\quad r=\left\{s_{1}, s_{2}, \ldots, s_{i}, \ldots, s_{K}\right\} \Rightarrow r \subseteq \mathbf{S}$ and a subsystem is a set of functions (or a subset of all functions) $s_{i}=\left\{f_{1}, f_{2}, \ldots, f_{j}, \ldots, f_{J}\right\} \Rightarrow s_{i} \subseteq \mathbf{F}$.

System component interaction (i.e. the interaction between humans and robots) plays an important role in the configuration of the system. To complete the system definition, the following list of axioms detail a base set of relationships that can exist among system components.

1. A function can be controlled by only one operator

2. An operator can control multiple functions

3. One task can occupy multiple subsystems

4. A single subsystem can concurrently execute multiple tasks

\section{SYSTEM COMPONENTS}

\subsection{Workload Estimation}

As stated, operator cognition serves to supplement autonomous control when intrinsic decision-making capabilities hinder expected robot action; however, human cognition is an exhaustible resource, which limits the scope of operator control [4]. Using the ACT-R cognitive architecture [4], human cognition can be modeled and estimated as a means of regulating operator control activity to prevent cognitive overloading. According to [5], cognition can be separated into six categories (e.g. working memory, longterm memory, vision, motor, speech, and audition.) that compose cognitive processes. Quantifying these cognitive areas produces a cognitive capacity vector $\left(\vec{\Lambda}=\left[\lambda_{w m}, \lambda_{l t m}, \lambda_{v}, \lambda_{s}, \lambda_{m}, \lambda_{a}\right]^{T}\right)$ that numerically describes the current/future cognitive status of an operator given the environmental feedback.

This formal representation of human cognition can be extended to represent the cognitive load induced from managing robot functions. The cognitive loading vector $\left(\vec{\Gamma}=\left[\varphi_{w m}, \varphi_{l t m}, \varphi_{v}, \varphi_{s}, \varphi_{m}, \varphi_{a}\right]^{T}\right)$ describes the cognitive capacity consumed in each cognitive area through function interaction. It is important to note that varying the level of function autonomy will alter the amount of cognitive capacity consumed; however, this matter will be addressed in the following sections.

\subsection{Workload Mitigation}


To enable robot functions the ability to adapt to environmental dynamics, the autonomy level controlling function behavior must also be dynamic. Adjustable autonomy et al [2], [3], and [7] provides a variable level of human-function interaction that mitigates the workload of operators and robots to the requirements of the system. Derived from [8], the four levels of function autonomy used in this work are:

1. Fully autonomous: periodic monitoring of function progress with infrequent, asynchronous operator interaction.

2. Semi-autonomous: minimal operator intervention with infrequent, yet sometimes critical, levels of operator interaction

3. Indirect Manual Control: discrete and periodic intervention with moderate to relatively high levels of operator interaction

4. Manual Control: continuous and direct intervention with consistently high (and perhaps maximal) levels of operator interaction

The cognitive load induced from a fully autonomous function (requiring little attention) will be less demanding than a manually controlled function (requiring constant attention) identifying a relationship between cognitive loading and functional autonomy. More formally, this relationship varies induced cognitive load as functional autonomy transitions from one level to another. To model the effects of autonomy transitions on cognitive loading, a set of linear transforms $\left(\Delta_{\mathbf{k}}\right.$ for $\mathrm{k}=1 \ldots 4$ autonomy levels) are established. Mathematically, this representation permits the occurrence of level-to-level autonomy transitions to dynamically recalculate the cognitive loads of the affiliated robot function.

\subsection{Workload Optimization}

As defined, operator-robot work systems can become extremely complex and intractable structures since multiple tasks are distributed over multiple functions and managed by multiple operators. Even then, dynamic adjustment of autonomy exponentially inflates the number of operatorfunction combinations creating a vast configuration space. The required solution will involve searching this configuration space to determine a preferable set of operator-functions matches.

To develop the search technique, the definitions from the previous sections are condensed and composed into an equation of matrices. Combining all cognitive capacity vectors into a capacity matrix $\left(\mathbf{H}_{\mathbf{C}}\right)$ and all cognitive loading vectors into a loading matrix $\left(\mathbf{R}_{\mathbf{C}}\right)$ the actual cognitive load of the system $\left(\mathbf{H}_{\mathbf{L}}\right)$ is determined by $\mathbf{R}_{\mathbf{C}} \mathbf{M}=\mathbf{H}_{\mathbf{L}}$ where $\mathbf{M}$ is Boolean configuration matrix. The role of workload optimization is to select an $\mathbf{M}$ that meets the following criteria: 
1. $\mathbf{H}_{\mathbf{L}}$ is minimized or at most $\mathbf{H}_{\mathbf{L}}<\mathbf{H}_{\mathbf{C}}$ for all matrix elements enforcing the cognitive load to be bounded by human cognitive capacity.

2. The number of managed functions (i.e. the number of rows in $\mathbf{M}$ that contain a non-zero element) is maximized enforcing that no functions are left unsupervised during task completion.

These constraints work in opposition to one another: minimization seeks to preserve cognitive capacity while maximization attempts to consume it. In situations where both conditions cannot be satisfied, a compromise must be established so that the system is optimally arranged to operate with maximum efficiency. This paper represents work efficiency as a system cost function that processes several system-critical parameters. These parameters include

1. Functional Priority: the relative importance of a function (e.g. highpriority functions should be given precedence when cognition is limited)

2. Preferred Configuration: the default configuration of operators and functions (e.g. the system should preserve this configuration whenever possible)

3. Authorization: the permission granting or denying an operator-function match (e.g. unauthorized matches should not exist)

4. System Anticipation: the elapsed time of a function/operator request for autonomy alteration (e.g. prevent cognitive starvations)

5. Task Inertia: the elapsed time between a linked operator and function (e.g. prevent unnecessary context switching among assignments)

By quantifying these parameters, the cost function can be tailored to suit the requirements of any work system and allow efficiency to be determined by comparing relative system cost. For example, the minimum system cost can be cast as the goal for searching the configuration space. The resulting configuration defined at the goal will be the system's M.

\section{THE OPTIMIZATION ALGORITHM}

This problem is easily seen to be NP-hard (by reduction from the binpacking problem [8]). As such, this paper utilizes an approximating algorithm based on The Transportation Algorithm ([9] and [10]) to determine system configuration. This algorithm is a well-documented optimization technique that has several similarities with the combinatorial issues of operator-function configuration. In summary, this algorithm determines a shipping network that maximizes the profits of a group of suppliers selling goods to a group of consumers such that the overall cost of 
shipping is minimized. Similarly, an optimal operator-function configuration is one where the number of functions receiving attention is maximized while the cognitive demand placed upon the human crew is minimized.

Despite the similarities, the Transportation Algorithm cannot perform workload optimization due to the following complications.

1. The Transportation Algorithm processes scalar quantities whereas cognitive capacity and cognitive loading are vector quantities.

2. A shipment matrix is composed of continuous, real number values whereas a configuration matrix contains discrete, Boolean values.

Complication (1). This situation requires modification to the vector components. As such, the cognitive capacity and loading vectors are collapsed into scalar components by selecting the smallest component from each $\vec{\Lambda}$ for operator representation and the largest component from each $\vec{\Gamma}$ for function representation. This reduction of dimensionality does affect the optimality of the configuration; however, the approximation drastically reduces the configuration space to improve the speed of computation.

Complication (2). This situation requires manipulation of continuous flow into discrete containers. To approximate an optimal configuration, the maximal flow component from each column of the configuration matrix is selected as the human-function match. Occasionally, selecting the maximal component can lead to cognitive overloading; however, if this case occurs, the work system varies the level of autonomy for any overloading functions and reprocesses the configuration.

It should be noted that these simplifications are only reasonable when the cognitive loading vectors are small relative to cognitive capacity vectors. When these vectors are on the same order of magnitude, the accuracy of approximation algorithm will degrade resulting in a systemic tendency to unnecessarily increase levels of functional autonomy.

\section{SIMULATIONS AND CONCLUSIONS}

To demonstrate the capabilities of adaptive operator-robot work systems, a simulation was devised using the mathematic model and algorithm stated in this paper. The operator-robot work system was defined as a combat scenario involving two UAVs, five UGVs, and three operators. Each robot was given a unique set of subsystems (and functions) while operators were provided varying cognitive capacities to emulate variable levels of task training and operator skill.

A cost function in the form of $\mathbf{C}=f\left(\mathbf{M}_{0}, i, j\right) \cdot e^{g\left(\mathbf{p}_{i}, \mathbf{q}_{\mathrm{i}}, \mathbf{Z}_{i j}\right)} \cdot\left(1+\alpha \mathbf{A}_{i j}\right)$ was selected for simulation where $\mathbf{C}$ is the cost for pairing the $i^{\text {th }}$ operator to the 
$\mathrm{j}^{\text {th }}$ function, $\mathbf{M}_{0}$ is the preferred configuration, $\mathbf{p}$ is the functional priority, $\mathbf{q}$ is the system anticipation, $\mathbf{Z}$ is the task inertia, and $\mathbf{A}$ is the authority. The function $f\left(^{*}\right)$ returns a constant that reflects the grouping assignment while $g(*)$ returns a composition of the priority, request time, and control inertia. This composition is placed into an exponential operator to give numerical importance to the mentioned parameters. Finally, $\alpha$ is selected to be significantly large such that when operator-robot parings are prohibited, $\mathbf{C}_{\mathbf{i j}}$ becomes extremely costly thereby effectively prohibiting the potential pairing.

The results in Table 1 were generated using two methods: (1) the aforementioned procedure and (2) static assignments where operators where permitted to control pre-assigned functions until their cognitive threshold was reached. Autonomy alterations were requested based upon a Gaussian distribution of problem occurrence for each function (Figure 2). Ten trials were performed with a simulation length of thirty iterations. The results are expressed as the average number of functions (AF), the unassigned function per iteration (UF), the unassigned functions with high priority per iteration (UFHP), total autonomy switches (TAS), and total context switches (TCS).

\begin{tabular}{cccccc}
\multicolumn{2}{c}{ Table 1. Simulation Results } \\
\hline AF & Method & UF & HPUF & TAS & TCS \\
\hline \multirow{2}{*}{8} & Optimized & 0.00 & 0.00 & 39.00 & 0 \\
& Static & 0.00 & 0.00 & 39.00 & NA \\
\multirow{2}{*}{31.3} & Optimized & 15.86 & 1.39 & 90.5 & 13 \\
& Static & 16.16 & 5.17 & 90.5 & NA
\end{tabular}

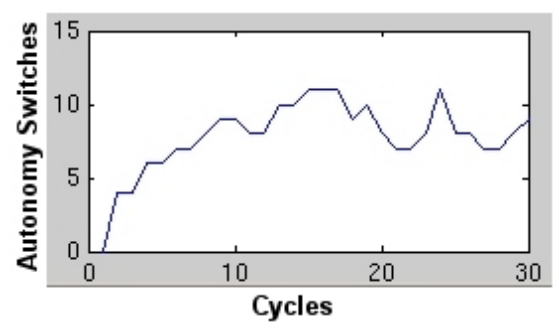

Figure 2. Autonomy level switching manifested simulations of the described scenario.

The first trial was a simple example demonstrating that systems with ample cognition have identical static and optimised results since operators were not cognitively overloaded in task management. The true benefits of the adaptive algorithm occur when the system becomes too complex for static assignments. As in the second trial, the adaptive model (on average) supervised 0.3 more functions than static assignment while also allowing 3.78 more top-priority functions to be managed at each cycle. Clearly, if top- 
priority functions were absolutely critical for task completion, then the optimized procedure would be the preferred implementation.

Finally, adaptive operator-robot work systems show great potential as a supervising control mechanism. For example, simulation shows a $372 \%$ increase of high-priority function supervision over static assignment for each cycle. The cumulative improvement in functional management can also be quite significant when evaluated across missions having hundreds of thousands of cycles. Furthermore, system simplification (while not providing a guaranteed optimal solution) yields an approximation that can be estimated in a small polynomial time (i.e. the time complexity of the Transportation Problem) and is more amenable to the real-time control cycles demanded for mission critical operation in unstructured environments

\section{References}

1. N. Jordan. (1963). "Allocation of functions between men and machines in automated systems," Journal of applied psychology, Vol 47, No. 3.

2. M. Montemerlo. (2000). "Uncertainty Based Sliding Autonomy," Doctorial Thesis Proposal. CMU Pittsburgh, PA.

3. D. Kortenkamp, Keirn-Schreckenghost, R. Peter Bonasso. (1991). “Adjustable Control Autonomy for Manned Space Flight Systems,” Proceedings of IEEE.

4. C. Lebiere, J. R. Anderson, D. Bothell. (2001). "Multi-tasking and cognitive workload in an ACT-R model of a simplified air traffic control task," Proceedings of the $10^{\text {th }}$ Conference on Computer Generated Forces and Behavior Representation. Norfolk, Va.

5. J.R. Anderson, C. Lebiere. (1998). The Atomic Components of Thought. Mahwah, NJ: Erlbaum.

6. K. S. Barber, C. E. Martin. (1999). "Specification, Measurement, and Adjustment of Agent Autonomy: Theory and Implementation. Autonomous Agents and Multi-Agent Systems," Autonomous Agents and Multi-Agent systems.

7. T. Fong, C. Thorpe, C. Baur. (2001). "Collaboration, Dialogue, and Human-Robot Interaction," $10^{\text {th }}$ International Symposium of Robotics Research, November 2001, Lorne, Victoria, Australia.

8. B. Korte, J. Vygen. (1991). Combinatorial Optimization: Theory and Algorithms. Springer. New York, NY.

9. O. Ore. (1962). Theory Of Graphs. American Mathematical Society, Providence.

10. G. Strang. (1986). Introduction to Applied Mathematics. Wellesley-Cambridge Press. Wellesley, MA. 